\title{
"I Wish I Could Do More": A Qualitative Meta-analysis of Early Career En- gineers' Perceptions of Agency in their Workplaces
}

\author{
Dr. Benjamin David Lutz, California Polytechnic State University, San Luis Obispo
}

Ben Lutz is an Assistant Professor of Mechanical Engineering Design at Cal Poly San Luis Obispo. His research interests include innovative pedagogies in engineering design, conceptual change and development, school-to-work transitions for new engineers, and efforts for inclusion and diversity within engineering. His current work explores how students describe their own learning in engineering design and how that learning supports transfer of learning from school into professional practice as well as exploring students' conceptions of diversity and its importance within engineering fields.

\section{Dr. Nathan E. Canney, CYS Structural Engineers Inc.}

Dr. Canney conducts research focused on engineering education, specifically the development of social responsibility in engineering students. Other areas of interest include ethics, service learning, and sustainability education. Dr. Canney received bachelors degrees in Civil Engineering and Mathematics from Seattle University, a masters in Civil Engineering from Stanford University with an emphasis on structural engineering, and a $\mathrm{PhD}$ in Civil Engineering from the University of Colorado Boulder.

\section{Dr. Samantha Ruth Brunhaver, Arizona State University}

Dr. Samantha R. Brunhaver is an Assistant Professor within The Polytechnic School, one of six schools in the Ira A. Fulton Schools of Engineering at Arizona State University. She is a mixed-methods researcher with focus on the preparation and pathways of engineering students. Her specific research interests include engineering student persistence and career decision-making, early career engineering practice, faculty pedagogical risk-taking, and entrepreneurial mindset. She completed her B.S. in Mechanical Engineering at Northeastern University and her M.S. and Ph.D. in Mechanical Engineering at Stanford University. Prior to ASU, she worked as an engineer at A. W. Chesterton, Boston Scientific, and Procter \& Gamble. 


\title{
"I wish I could do more": A qualitative meta-analysis of early career engineers' perceptions of agency in their workplaces
}

\begin{abstract}
Engineering students graduate from their programs with a broad range of skills that are set by professional societies, industry recommendations, and other stakeholders in student success. But when those engineers enter their jobs, how are those skills utilized and nurtured by the organizations they enter? The purpose of this paper is to present a cross-sectional, secondary qualitative analysis of research exploring the experiences of recent engineering graduates as they move from student to professional. Of particular interest were the ways engineers describe their autonomy or sense of choice, the way engineers recognize and make sense of their organizations' values, and the alignment (or lack thereof) between personal values and those of their organization. To do so, qualitative data sets from three different studies of engineers' experiences at various stages in their professional trajectories were combined and thematically analyzed, producing four major themes that speak to the ways engineers perceive their sense of agency in their work experiences. Looking across data sets, themes emerged regarding empowerment, organizational fit, and workplace expectations. While these themes were common across the studies included in the analysis, the way the themes manifested across data sets raises interesting questions about the formation of engineers and the socialization experiences that contribute to that formation. As research on engineering practice continues to develop, it is important that researchers consider where engineers are within their career trajectory and how that influences their perceptions about the work they do and the agency they have within organizations.
\end{abstract}

\section{Introduction and Background}

What constitutes engineering practice is constantly changing and revised as engineering problems become more complex and multidisciplinary, and the scale on which engineering problems are solved increases [1]. Numerous blue-ribbon reports and recommendations from engineering professional societies have made calls to name and develop the skills engineers need for success in their careers. For example, in The Engineer of 2020, the National Academy of Engineering (NAE) asserts that engineering graduates will need skills such as practical ingenuity, high ethical standards, strong communication, and leadership [1-2]. Further, in their "Vision 2030", the American Society of Mechanical Engineering (ASME) reports that engineers need to develop more innovation and creativity, a higher standard of professional skills, and more practice-based engineering experiences [3]. Beyond the technical knowledge required to address these complex problems, the American Society of Civil Engineers (ASCE) set out in their second edition body of knowledge that engineers should adopt attitudes of curiosity, entrepreneurship, fairness, respect, sensitivity, thoughtfulness, and tolerance [4]. The broad range of expectations and abilities needed for engineers to adequately address tomorrow's issues require not only a diverse approach to educating engineers, but will also require work places that continue the process of broad development in early career employees.

Industry representatives have echoed these issues, noting current graduates have weaknesses in practical experience, understanding of engineering codes and standards, leadership skills, and 
knowledge of business processes, to name a few [5]. More recently, ABET has revised their accreditation criteria to align more closely with these calls and address knowledge and skills gaps between school and professional practice [6]. These reports, recommendations, and accreditation criteria are important for engineering education because they help guide curricular choices about what students should learn and inform decisions about what kind of experiences to provide.

To be sure, the skills noted above are valuable traits for engineers, but when engineers actually enter and work in engineering organizations, how do those skills develop? Engineering practice is complex, multifaceted, and dependent on a range of social, organizational, and technical constraints. As a result, how engineering is practiced and what constitutes authentic practice can vary both within and across organizations [7-10]. For some engineers, practice might entail conducting analysis and testing on components of a particular design; for others, it might look more like managing the range of tests that are conducted throughout product development [11]. Nonetheless, engineering practice can comprise a wide range of activities and accompanying skills, and these might be different from one organization — or job - to the next.

The purpose of this paper is to explore the interaction between engineers' experiences in authentic practice and the way their organizations facilitate or hinder the development of skills and dispositions noted as vital in reports and accreditation criteria. If engineering graduates have the skills called for by professional societies and accreditation bodies, it seems important that the organizations that employ these engineers provide spaces to nurture and grow such skills. To that end, we pose the following research questions:

\section{RQ1. How do workplace environments enable or constrain early career engineers' ability to apply their skills developed as undergraduates? \\ RQ2. How do early career engineers recognize and reconcile misalignments between their goals, values, and skills and those required to do their jobs?}

To explore these questions, we present a thematic analysis [12] of three different data sets of engineering practice with engineers at various points within their careers. The first data set examines the perspectives of engineering juniors and seniors having recently completed a co-op or internship, the second investigates the experiences of recent engineering graduates during the first twelve weeks of their jobs, and the third explores the beliefs of more experienced engineers who engaged in service-oriented projects as students. We selected these data because they both represent three different points in time and speak to different levels of understanding of and familiarity with engineering organizations. We conducted a thematic analysis to identify major themes related to engineers' agency and the way their organizational structure interacts with their own beliefs and skills.

Specifically, we explore themes of Relational Empowerment, Structural Empowerment, Organizational Fit, and Expectations. Results suggest that the point at which one is in their engineering career (i.e., new vs. experienced) might influence how they experience these themes, and point to critical questions surrounding the role of professional preparation from engineering programs and organizations. 


\section{Methods}

\section{Context}

This paper presents a preliminary study looking across different data sets for commonalities in the experience of engineers in professional practice. Motivation for this particular paper was catalyzed by interactions at the NSF-sponsored Research on Engineering Practice (REP) workshop at Santa Clara University in October 2018 (NSF grant numbers 1832966, 1833055, 1833113, and 18331246). The authors met during a brainstorming session at the workshop that was designed to generate ideas for publications, proposals, and other potential research collaborations. Discussions centered on a common interest in better understanding how engineers' own beliefs, values, and goals interact and align (or do not align) with those of the organization they work for. As a first step in exploring these ideas, we shared the content of existing data we each collected with practicing engineers at different points in their career trajectories.

The purpose of this research, then, was twofold. First, based on the common interests of the authors, we wanted to share ideas and spark discussion around the relationship between identity, socialization, and organizational culture within engineering. Second, we wanted to examine the presence of these themes at different points in an engineering career. As part of a larger effort to explore the experiences of practicing engineers, we focused on the ways that engineers talk about their own agency or autonomy as it concerns the work they do and the organization they work for. We conducted a thematic analysis across three independent data sets and identified emergent themes. The following sections give an overview of the three complementary data sets used in the present work, describe the thematic analysis process, and offer potential limitations for consideration when interpreting our findings.

\section{Data Collection}

As noted, this research presents findings from three different data sets exploring the experiences of engineers in practice. None of the data sets were collected with the explicit purpose of exploring the themes noted below, but through discussions at the REP workshop, the authors converged on an approach to synthesize and look across different kinds of data to probe for similarities and differences. The result is a secondary analysis [13] that investigates salient elements of engineering practice as well as the ways those might vary according to differences in samples and research approaches. All participants were given pseudonyms prior to analysis.

\section{Data Set 1}

The first data set was comprised of semi-structured interviews with juniors and seniors at six engineering schools across the country. These interviews were part of a longitudinal, mixedmethods study exploring engineering students' beliefs and experiences regarding their job search and career decision-making processes. A survey was administered to approximately two thousand engineering students at the six schools in the fall of their junior or senior year. Sixtytwo students were interviewed between two and five months later, purposefully sampling for mechanical engineering and chemical engineering students (to limit potential differences in the data by discipline) and to match (to the extent possible) the student demographics in each department. Students determined to be actively looking for their first job post-graduation were interviewed using one version of the interview protocol, while all other students were 
interviewed using another. Each interview protocol covered questions about the student's postgraduation career plans and job search related activities. If the student had professional engineering co-op or internship experiences, they were asked to describe what these experiences were like and how they may be informing students' career-related thinking going forward. Each interview was approximately 45 minutes long, conducted by phone, recorded, and transcribed. Further details about the overall study and the interview data collection process are provided in [14] and [15]. Because the current analysis focuses on experiences within engineering organizations, the data set includes interviews with students who reported having engineering coop or internship experiences, only.

\section{Data Set 2}

The second set of data was collected as part of a longitudinal, multi-case study of the school-towork transition for recent mechanical engineering graduates. Twelve participants were recruited before graduation and followed through the first twelve weeks of their new jobs. Mechanical engineering was selected because of the significant industry focus of the major as well as the size of the program at the study university as well as others across the country. For a full discussion of the recruitment and sampling approach, see [11].

Data was collected in three phases. First, participants were interviewed before they graduated. These interviews were semi-structured and explored students' expectations about their jobs, the skills they needed in their jobs, the skills they had gained from their undergraduate experiences, and how prepared they felt to enter their jobs. Second, participants responded to weekly openended journal prompts in which they unpacked a significant challenge, accomplishment, or realization. Questions were designed to ask participants to describe the specific event, the involvement of others, and their perceived learning as a result of the event. Third, participants were interviewed at the end of the twelve-week journaling period. The interviews were again semi-structured and designed to bring closure to the experience. During the third phase, interview questions focused on participant reflections of their experience in the school-to-work transition and also provided space for them to discuss additional significant learning not captured in the journals and elaborate on particularly significant events. Because the present work focuses on experiences within engineering organizations, the data set is comprised of journal entries from phase 2 and reflective follow-up interviews in phase 3.

\section{Data Set 3}

The third data set came from interviews with engineering professionals who had been active in some form of engineering service (e.g. Engineers Without Borders, Peace Corps/Masters programs) as undergraduate or graduate students. Nineteen alumni were interviewed using a semi-structured interview protocol focused on their career pathways and the impacts of their service experiences on their career trajectories. Interviews lasted between 30 and 90 minutes and were recorded and transcribed for analysis. The participants had a wide range of time in professional capacities, from one to ten years. The majority of the participants were still in some form of engineering job, though two had left engineering for professions that they felt were more service oriented $-\mathrm{K}-12$ teaching and family therapy. The other seventeen participants represented traditional engineering consulting jobs as well as non-profits focused on conservation, international development, and public policy. Some participants had continued to engage in forms of engineering service as working professionals, while others did not. These 
interviews were used in an exploratory sequential mixed-methods approach to develop a survey which was distributed more widely. Results from analysis of the interviews and survey responses pertaining to service and social responsibility can be found in [16] and [17], respectively.

\section{Data Analysis}

\section{Thematic Analysis}

The purpose of the analysis was to explore themes in three different data sets to better understand the salience of particular issues across a range of engineering practice settings and career stages. To do so, we applied thematic analysis recommendations by [12], following a six step process to arrive at final themes.

\section{Familiarize with data \\ 2. Generate initial codes \\ 3. Search for themes \\ 4. Review themes \\ 5. Define and name themes \\ 6. Produce the final report}

Though these steps are presented as a linear process, it is important to note that the analysis was iterative as the authors moved back and forth between steps to revise, redefine, and modify our themes throughout the process.

First, we met to familiarize ourselves with each other's data. As noted, the data in the present analysis were collected at different time points, from different populations, and for different initial purposes. To do so, we provided each other with select passages that highlighted or were related to our guiding research questions. For example, first author Lutz asked questions specifically related to participant expectations, and excerpts from the answer to that question were combined, shared, and discussed with the research team. Second, we generated initial codes. We focused on developing descriptive codes that highlighted issues of agency in and alignment with an organizational structure. These initial codes were then organized into conceptually similar groups to develop themes (step 3). This process led to the generation of four unique themes within the data.

In step four, we reviewed these themes for internal consistency and clear conceptual boundaries. We ensured that passages within a given theme referred to similar concepts and ideas, while at the same time looking across themes to verify that each theme was conceptually distinct. In this step, we made sure that all passages coded as Relational Empowerment referred to similar ideas, while also checking to ensure that all excerpts coded as Relational Empowerment were different from those coded as Structural Empowerment, for example. By establishing internal consistency as well as delineating clear conceptual boundaries, we were able to determine why a particular passage fit in one theme as well as why it did not belong to others. Fifth, given our final list of themes and accompanying coded segments, we created names and operationalized definitions for each theme that emerged from our analysis. Finally, to produce the report, we collaborated to write the present work. The resultant process produced a set of four themes that emerged from analysis looking across three different data sets. 


\section{Limitations}

A few limitations should be considered in the context of the present work. First, the analysis presented was secondary to the original study designs. Data Set 1 was collected to explore the job search and career decision-making processes of engineering juniors and seniors. Data Set 2 was collected to explore the experiences of recent mechanical engineering graduates during the school-to-work transitions. Data Set 3 was collected as part of a study investigating the experiences of engineering professionals who were engaged in service-based activities either through their employers or some other professional organization. As a result, the data and findings presented in this paper are tangential to the initial intent of each project. However, each set of data describes the experience of engineers and their interactions with organizational structures, cultures, and norms and thus provide interesting insight when looking across them.

Second, and relatedly, the participants in each data set represent engineers at rather different points along their career trajectories. Engineers who are just entering an organization to work in a job or internship are likely to experience their organization much differently than those who have been practicing professionals for more extended periods of time. One consequence of this difference in experience appears to be some level of distortion in the reflective experiences of participants in Data Set 3. For example, as noted below, where engineers from each data set described settings with ambiguity and uncertainty, participants with more experience tended to recall their early work experiences as being more within their control and influence. It is therefore possible that as participants look back on prior experiences, their current perceptions influence the way they recall earlier organizational interactions. That is, experienced participants with more autonomy in their current positions might project that sense of empowerment onto their earlier career in ways that distort their actual lived experiences. Nonetheless, when looking across participants and data sets, similar themes emerged in our findings and point to the salience of the findings for understanding the experiences of engineers in practice.

\section{Results}

Based on iterative conversations between the authors prior to and during coding, the following four themes were identified: Relational Empowerment, Structural Empowerment, Expectations, and Fit. Both Relational Empowerment and Structural Empowerment speak to participant expressions around experiences of autonomy and support (or lack thereof) in their jobs and primarily address our first research question. Relational Empowerment refers to ways in which coworker and supervisor interactions contributed to participant feelings of being empowered or disempowered as early career engineers. Structural Empowerment captured comments from participants that pointed to an awareness of the corporate structure that they were engaging in while working. These coded segments focused on either confusing aspects of corporate culture, barriers experienced by participants, or lessons navigating the corporate culture in order to achieve personal goals. These first two codes aligned with the first research question focused on skills development and workplace environment.

The next two themes focused on the second research question, addressing issues of alignment or misalignment between participants' goals, values, and skills and professional experiences. 
Expectations applied to conversations from the three data sets around participants' thoughts about what the nature of their work would be like. Fit was used to capture expressions that focused more on the compatibility between participants' personal elements (desires, goals, values, ethics, etc.) and those of their organization.

An overview of these emergent themes and some examples are provided in Table 1, and each theme is described in more detail below. Across the four themes, recollections were more "in the moment" for participants in Data Sets 1 and 2, while they were retrospective for participants from Data Set 3, who may have been in engineering roles for up to a decade. There was also considerable overlap and interplay between codes. Observations around the differences between data sets and the overlap between codes will be further explored in the discussion section.

Table 1: Overview of emergent themes across data sets.

\begin{tabular}{|c|c|c|}
\hline Emergent Theme & Definition & Examples \\
\hline $\begin{array}{l}\text { Relational } \\
\text { Empowerment }\end{array}$ & $\begin{array}{l}\text { Descriptions of work interactions } \\
\text { that made participants feel a sense } \\
\text { (or lack) of autonomy or agency in } \\
\text { their jobs }\end{array}$ & $\begin{array}{l}\text {-Having control over work } \\
\text { decisions } \\
\text {-Feeling or being in control of } \\
\text { tasks and work assignments }\end{array}$ \\
\hline $\begin{array}{l}\text { Structural } \\
\text { Empowerment }\end{array}$ & $\begin{array}{l}\text { Perceptions of corporate } \\
\text { organization/hierarchies that made } \\
\text { participants feel a sense (or lack) of } \\
\text { autonomy or agency in their jobs }\end{array}$ & $\begin{array}{l}\text {-Learning how to operate within a } \\
\text { work unit or team } \\
\text {-Leveraging corporate structure to } \\
\text { achieve particular outcomes }\end{array}$ \\
\hline Expectations & $\begin{array}{l}\text { Descriptions of the work participants } \\
\text { expected to be doing or thought was } \\
\text { possible within the organizations }\end{array}$ & $\begin{array}{l}\text {-Discussion of potential work roles } \\
\text { or assignments } \\
\text {-Beliefs about what kind of work } \\
\text { participants should be doing }\end{array}$ \\
\hline Fit & $\begin{array}{l}\text { Perceptions of compatibility between } \\
\text { organizational goals and values and } \\
\text { those of the participant }\end{array}$ & $\begin{array}{l}\text {-Tension/alignment between } \\
\text { organizational mission or work } \\
\text { assignments and participants' } \\
\text { personal goals and values } \\
\text { - Finding locations within an } \\
\text { organization that align with } \\
\text { participant values }\end{array}$ \\
\hline
\end{tabular}

\section{Relational Empowerment}

Segments attributed to this theme focused on participant recollections of work interactions that either enabled or restricted autonomy in their professional work. Participants from the three data sets talked about relational empowerment in different ways given their differing tenure in their positions. One participant from the second data set with recent graduates said the following:

... I feel like a useful team member at least in the way of helping new hires get up to speed. I've been given more meaningful tasks, but I feel like I'm still not solely responsible for one thing, be it a part or process or something like that. I am 
hoping to succeed with the supporting tasks I've been given and prove myself to be able to handle more responsibility and "own" something. I've also had a few moments where my boss has complimented me or my work and that has been very rewarding and made me feel hopeful about my future and my success in my career. I feel like my boss trusts me to be successful in the tasks he gives me and trusts me to ask for help when needed, speak up when I feel overloaded, and do my work without needing him watching over my shoulder. I still firmly believe his confidence in my ability is related to the experiences I've had as a coop/intern in the past, whether that is due to actual skills gained or my own confidenceprobably a good mix of both. - Eric, Data Set 2

For this participant, there is a clear understanding that he is not in charge of what work he does. "I've been given more meaningful tasks..." speaks to the notion that tasks come from elsewhere and are given to him. Eric believes that if he is able to "prove [him] self" then he will eventually be given autonomy to set his own agenda ("own something").

At the same time, Eric's response shows that he feels empowered to ask questions and to advocate for himself in his job ("I feel like my boss...trusts me to ask for help when needed, speak up when I feel overwhelmed, and do my work without needing him watching over my shoulder"). Eric not only feels empowered to ask questions, but also recognizes that questions are an integral part of being a new engineer and growing in his new role. Moreover, Eric feels a sense of autonomy in that he is able to do his work "without needing him watching over my shoulder." This sets up an interesting dynamic between the need for new employees to ask questions, but also to not feel like their boss is looking over their shoulder. There seems to be a balance, then, where an employee who does not ask questions and wastes time may need more oversight, while an employee who feels empowered to ask questions may not require as close oversight and therefore grows in their feelings of empowerment.

Eric also sees autonomy in terms of "trust" from his boss. He attributes this trust to his past performance, but also to the degree of confidence that he projects, which he attributes to his past co-op/intern experiences. This brings up an interesting notion, where autonomy may not always be related to performance, but may also be related to projections of confidence. Eric points to compliments from his boss as support for, and reinforcing his own confidence in his performance. While it is not surprising that a new employee less than twelve weeks into a job would not be setting their own agenda, this theme was also observed among the other data sets.

From the third data set, one participant described their gender and age as impacting their feelings of autonomy and empowerment in their job, saying:

...it's challenging more because, again, you have the grumpy old sub-contractor or superintendents who have been doing this for 40 years, and they don't want to listen to you, a 20-something girl. Let's be frank, like they don't want to listen to the 20-something woman, like come on. And so I was really interested in that aspect [sustainability] ... So then I was really knocking on the [door] and was like, "You have a hole there, I can fill that hole." And they're like, "You need more experience, she was the director, like we can't make you director of 
sustainability." But I kind of, like nudged them enough, I don't know what my title was, but they made me a sustainability engineer. Everybody called me the

director. I was like, thanks. That's not what my business card says but it's okay. Ella, Data Set 3

In this quote, Ella points initially to a lack of empowerment from external sources (the "grumpy old sub-contractor or superintendents who have been doing this for 40 years") and, in this dynamic where she does not feel supported by upper management, she characterizes herself as " $a$ 20-something girl", though she later refers to herself as a "20-something woman." It is interesting how Ella labels herself not only by her age, but as a "girl" (which contradicts the age, where a "20-something" female is factually a woman). This labeling could point to feelings that Ella had in this situation of disempowerment and residing in a "less-than" position in comparison to the sub-contractors or superintendents. Despite being in what appears to be an un-empowered position, Ella persists and, due to her determination ("I kind of like nudged them enough"), she is able to create a position and work on projects that she wants. It is unclear from this quote how long it took Ella to achieve that goal, but she was eventually able to create a niche for herself through her own self-advocacy.

Comparing the experiences of Eric and Ella, positive feedback from a supervisor affords Eric with a sense of agency and confidence about his work, whereas lack of acceptance by senior colleagues, at least first, appears to shake Ella's confidence and put her in a less-than-favorable position of having to fight for what she wants. This connection between confidence, autonomy, and feelings of success in the corporate structure mirrors what Stevens et al. [18] found around students' ability to navigate academic structures as a key component of their success in engineering education.

Even more importantly, the quotes from Eric and Ella represent key distinctions seen in the experiences of men and women across all three data sets; namely, that women engineering students and recent graduates experience fewer instances of support and encouragement from managers and coworkers in the workplace than their male counterparts do. Put another way, women were less able than men to identify "sponsors" [19] and less likely to report feelings of self-efficacy and connectedness at work in every data set comprising this study. Such discrepancies raise questions such as, who wants to, gets to, and continues to be an engineer in the current climate, and how much do engineering employers recognize and support the important perspectives that diverse individuals bring? From the juxtaposition of Eric and Ella's stories, it is clear that more work is needed to help women and other underrepresented groups feel welcome and included.

\section{Structural Empowerment}

In contrast to relational empowerment, structural empowerment represents the ways in which the configuration of an organization or work group might enable or constrain engineers' sense of autonomy. Participants from the first two data sets spoke about corporate structure as they experienced and negotiated it at their first jobs post-graduation or their student coops/internships. On the other hand, participants from the third data set had more experience and were able to speak about corporate structures more holistically and even to compare one system to another if they had worked in several positions. This provided useful insight into how 
participants perceived corporate structures as contributing to or inhibiting a sense of personal and professional empowerment within their workplace environment. The following excerpt came from a participant in the first data set:

I had interviews with other companies that I was interested in ... but the decision making that went into picking that company was their focus on the individual employee because I had worked for bigger companies before like [Company] and what I found was that it wasn't personal, so I wouldn't be able to see all the people that are above me within the company, communicate with them on a, not [an] informal basis, but on a more regular basis than there was time available. This company is smaller, which gives me the freedom to do that more and also to be able to be recognized more easily for the work I do. - Ryan, Data Set 1

Here Ryan, an engineering senior, explains his reasons for accepting a job offer to work at a particular company. He compares this company to others at which he has had internships, describing these former places of employment as large, with complex organizational structures that made it difficult to network with "people who [were] above [him]" on a regular basis. By contrast, he calls the organization he will join after graduation smaller, with a more open corporate structure that he expects will afford him greater access ("the freedom to do that more") and greater visibility ("to be able to be recognized more easily") to upper management. Ryan's sentiments echo many participants in the first data set who eschewed jobs with multiple layers of bureaucracy for those in which they could start contributing and advancing immediately.

A participant from the third data set spoke of a different kind of freedom at work:

I mean, it's definitely a great job, and part of the reason why it's so great is that the people, that the owner of the company really just gets that you need to have a work-life balance, either you want to go back-packing and you want to be outside, that you also want to make an impact. So, the whole structure of, like our pay structure and our vacation policies are all designed so that people feel like they can take time off and work as much or as little as they want. - Jessica, Data Set 3

Jessica points to the flexibility of her corporate culture as being an important element of her satisfaction there. For her, having an owner who recognizes the "need to have a work-life balance" is critical for her feeling supported in this environment. She experiences support for having a work-life balance through alternative pay and vacation structures that allow flexibility ("so that people feel like they can take time off and work as much or as little as they want") and enable her "to make an impact". A similar way that other participants from the third data set mentioned corporate support through pay and vacation structures was when participants talked about their companies giving them an extra week of vacation every year if they used that week for an international service trip like an Engineering Without Borders build.

Another participant from the third data set, Philip, disclosed deeper struggles with the corporate structures, sharing the following:

\section{...I guess you always know that corporations make decisions based on the bottom}


line, right? I mean, that's number one. They may say things differently, but, you know, in the end that's...they all have to reply that they're...to act according to their stock broker's wishes [but] you know, what do you do when...like, for instance, you've got a groundwater plume and it's degrading into, like vinyl chloride, right? It's pretty nasty, it's the biggest risk in a chlorinated solvent plume usually, and it's most often a driver for action, you know... But you don't really have an understanding of where it is in the ground and what influences it to move down gradient and it's the stuff that's way down gradient. I mean [that] you're going to put more holes in the ground doesn't necessarily mean that you're going to understand anything any better... you don't know if that's gonna give you better information...or [if] it's gonna give you more information, you don't know if that information is gonna give you a better understanding of what's going on. And you have to take that into consideration when you're spending your client's money because it does influence their bottom line and it does. You know, if all things were great and ethics were the only thing you thought about, yeah, man, punch that place full of holes and do it in an environmentally sensitive way that you get the information you need, it doesn't matter how many you get. But ethics plays into that, you know? - Philip, Data Set 3

In this excerpt, Philip is struggling with his work as an engineer, his desire to do the right thing ("ethics plays into that") and to make informed decisions, with an understanding of the corporate structure as primarily being for profit ("corporations make decisions based on the bottom line... that's number one"). From this, and similar examples, one understanding of the corporate structure that participants expressed was that the business side of the industry trumped their engineering professional responsibilities to the public. Philip talked about weighing his need for more data about the groundwater plume with "spending your client's money." He sets up this conflict as an ethical issue, seemingly stating that he has to walk a tightrope by making the best decisions he can with the least amount of information so as not to spend the client's money.

This brings up interesting questions around who within organizations gets to decide what enough information is. Where is the line between an engineer's best judgement and the financial interests of the firm and their clients? What guidance do engineers have when those two things come into conflict? While Jessica experienced corporate structures that helped her feel empowered, able to take care of herself and achieve a work-life balance, Philip experienced elements of his corporate structure that could compromise his ability to act responsibility as an engineer, violate his personal and professional ethics, and cause him to feel disempowered.

\section{Expectations}

Participants from the second data set were asked directly about differences between their expectations for their work and their experiences twelve weeks into their jobs. One participant responded with the following:

I came to this job with kind of an open mind. I didn't really know what exactly I wanted to do after college, so I pretty much took the mentality of I was up for anything, job-wise. And this was kind of the first and best option that came around to me so I took it. I didn't really know what to expect, but from what my 
professors told me, just [the] engineering professional world in general is that you have to, like communicating with people that aren't engineers is a big thing and, you know, it certainly is in my job. - Jeff, Data Set 2

In this quote, Jeff talks about having not really known what to expect upon started his first job post-graduation and so, as a result, actively works to be open and flexible; in other words, to have few expectations. This uncertainty paralleled what many other participants from the second data set described as not knowing what projects they would be working on, not knowing what professional career trajectories within their company would be like, or, for some, believing they would be doing one thing when they took the job only to realize they would be doing something different on their first day. Some participants drew from previous co-op or internship opportunities as a guide, but, in general, having few, loosely held expectations seemed to be the most common strategy to negotiate the transition.

Jeff also draws from what "my professors told $m e$ " to inform his expectations. While it is not surprising that faculty are a key component in preparing engineering students for the professional world, it raises important questions about the messages faculty are presenting. How accurate are the messages that professors present regarding professional engineering? How many professors have personally experienced professional work, and does this impact their messaging? Are messages consistent across faculty? In Jeff's case, the message around the importance of communication with non-engineers matched his professional experience.

Other participants compared expectations set up by their schooling and experiences with professional work. One participant from Data Set 2 commented that he expected to do more technical work: "I thought I would be doing more technical work. I haven't done much technical stuff at all." This participant went on to talk about how a lot of his work was dealing with finances and project management. Another reported expecting more oversight of his work, saying "... [You are] basically by yourself and maybe one person might look after [your work]. So, yeah, I just have more responsibility than what I had before." This participant translated the lack of oversight over his work into a need for greater personal responsibility. This experience could be contrasted with the academic setting where, through grading or intensive mentoring of project-based learning, faculty again shape student expectations, this time, that someone else will always look over their work, relieving them of the responsibility to thoroughly check it before submitting.

The third data set provided a different perspective on Expectations than the second since most of those participants had been out of school for several years. Many of these participants reported having more difficulty finding jobs that paralleled their collegiate service backgrounds than they expected to. One participant, in talking about how his service experience influence his expectations, said:

Well, so, I loved what I had done with EWB. I mean, I did an undergrad (degree) that was pretty unrelated to EWB and, so, arguably that's probably where I was more competitive, in work was doing ground water (reclamation) at super fund sites, but it wasn't what I wanted to do. So, I was looking for anything that would kind of get me doing environmental engineering, you know, water projects with 
some relation to resource settings, ideally [in] developing countries. I didn't find employment in that right away, those jobs, you know, they all say entry-level position, meaning we hope you had six years of experience and a graduate degree. - Thomas, Data Set 3

Once again, Thomas' expectations were strongly influenced by his collegiate experiences, and specifically, his engagement with Engineers Without Borders (EWB). Similar to participants from the second data set, Thomas's experience was that the professional opportunities available to him were very different from what he had expected based on his collegiate experiences. This was a common theme for many participants in the third data set where expectations of being able to do development or service work as an integrated part of their engineering careers usually did not come to pass. Granted, this is a specific sub-set of students - those who were active in engineering service - but it parallels the experiences of the participants from the second data set where academic experiences set up expectations that may have been misaligned with professional realities.

\section{Fit}

While Expectations focused primarily on participant experiences of work, Fit focuses on issues of alignment between participant's personal elements (desires, values, goals, ethics, etc.) and the corporate culture they experience. In all three data sets, there were examples of positive and negative fit, or alignment and misalignment, between participants and their organization. One participant from the first data set said the following, evidencing positive fit:
For me, personally, I don't really mind the work I do so much. I don't value that as high priority. I more importantly value the people I'm working with and the company culture. When I was looking for jobs toward the end of summer and early fall semester, I was really just taking a look at different companies and how they treat their people and how they treat their customers, and for [Company's] case, their patients, people that depend on their medicine. I really value that they have a direct impact to the patients. I thought that was great. Then...I interned at [Company] over the summer, and I worked in the same group, the utilities engineering group. Apparently I did well enough to get a return offer, but more importantly, I enjoyed the people I worked with. They were always helpful and insightful whenever I had any questions. They never seemed annoyed by all my intern questions. - Chris, Data Set 1

In this quote, Chris unpacks his decision to take a full-time position with the company at which he interned. First noting that he places less emphasis on the type of work he does than on more social and cultural concerns, Chris recalls being impressed with having both coworkers who were receptive and helpful ("They never seemed annoyed by all my questions") and an organization that cares about the well-being of its people and customers ("I really value that they have a direct impact to their patients"). Chris's response also seems to imply that, although he did well enough at his internship to be asked to return, it was these larger concerns rather than the mere presence of a job offer that convinced him to accept. Indeed, perceived fit between individual values and organizational culture emerged as a positive contributor to early career choices in all three data sets, as demonstrated again in this next example: 
Sarah: ...I really maintained my interest in that type of work, you know, sustainability, community efforts. So I'm doing green infrastructure for storm water management with a company that is very, very interested in the concepts of community and sustainability. They have several large programs that are fundraisers for Water for People and EWB. It's kind of a core value of the company and I am so thrilled to be a part of it... I would say that those concepts are just, it's sort of a core part of my life...

Interviewer: So, when you say 'those concepts,' can you name those?

Sarah: Yeah, I would say for me it's the ideas of service to my community and helping communities in general improve their quality of life. The idea of sustainability is really a big deal to me. Social justice, I mean, so I, you know, I grew up Catholic, went to a Jesuit university. Sort of, those ideas, the social justice and, you know, service ideas are kind of drilled in but I think pretty valuable, so...But beyond that, like if I think back to telling people about how excited I was about this job, it was partly to do with being able to use my expertise and, you know, really, they wanted someone to come in and become a water quality expert for this office. And they wanted me. And that was really cool. But beyond that, the second thing I told people was how much they support these, you know, community and international development programs and that, I think that was probably a huge factor for me. - Sarah, Data Set 3

Here Sarah is describing not only an understanding of the corporate ethos around service and sustainability ("it's kind of a core value of the company"), but also speaking to her personal values around the same topics ("those concepts are just, it's sort of a core part of my life") and the alignment between the two. For Sarah, the compatibility between her personal values and those of her company was on par with her passion for the technical aspect of her job which she also enjoyed. If faculty and advisors only focus on alignment between students and the technical or financial aspects of an engineering position, could they be doing a disservice to students in the long run, especially for students with strong personal values and goals? How common is is that engineering students have the maturity and introspection to know what company values they are looking for and how to find those in a job? It would be interesting to see how many engineering graduates have a similar level of self-knowledge and advocacy to what both Chris and Sarah had.

Sam, another participant from the third data set, had a different experience at his job, noting:

I did get a job doing that in California with [Company], and I was there for about two years, and that was a pretty frustrating experience for me partly because I think the particular office I was in was pretty bad in terms of, like office dynamics and politics... Also, I think in terms of, like the way that our society and economics (from that standpoint), that there's a fundamental incompatibility that's being for profit and being for the public good (that I ran into) there, and so it would come to things like, I would be trying to argue for my clients doing less work in terms of remediating a chemical spill that they're responsible for or 
things like that where I didn't necessarily think that was in the public interest but it was in both the interest of their...their client's bottom line and also our bottom line to be able to keep clients and keep building and those kinds of things, and so there were a few situations where I was asked to do things that I thought were unethical, and I kind of asked around to other people in the field who had been in similar situations where, like the major client would say like, "Oh, we don't think that these laws are accurate. Can you go back and, like make these changes..."Sam, Data Set 3

Sam went on to describe instances where he was asked to bury information in report text rather than in diagrams to increase the likelihood that authorities would miss it. Sam left engineering and transitioned into family therapy where he felt that he could more easily achieve the positive impact on the world that he desired.

Similar to Philip's quote earlier, Sam expressed concern about experiencing "a fundamental incompatibility that's being for profit and being for the public good." For both of these participants, they saw the purpose of their engineering careers as "being for the public good" but experienced misalignment between that personal goal and their work as engineers. For Sam, this conflict manifested when his professional opinion on the proper corrective approach to a chemical spill was "in the public interest" but would cost their client too much money, potentially losing their business. He felt divided between his personal and professional obligations to hold paramount both the public safety and his company's ambitions with a big client. As an engineer in his first two years, perhaps Sam did not feel empowered enough to bring up this concern within his company. Additionally, the corporate structure at his firm seemed to be unstable, perhaps also threatening his feelings of empowerment from within the system, ultimately leading to his departure from the profession altogether.

\section{Discussion}

Situation in blue ribbon and national reports

The impetus for this paper was to examine, through secondary analysis of qualitative data, how characteristics which the blue ribbon and national reports call for future engineers to have align (or fail to align) with engineers' professional experiences. The broad range of skills and dispositions highlighted in these reports has been characterized as attributes of the "renaissance engineer" [20-21], oftentimes including a need for increased innovation, creativity, and entrepreneurship. The manifestation of these skills in the workplace, however, would seem to require significant empowerment and autonomy within employees. Additionally, creating an environment where engineers feel safe to take risks - which innovation and creativity certainly require - necessitates an atmosphere where vulnerability, imperfection, and mistakes are all accepted, if not encouraged [22].

Analyzing these three disparate data sets around themes of Relational Empowerment, Structural Empowerment, Expectations, and Fit has shown that, consistent with prior literature, early career engineers desire meaningful work, autonomy, and connectedness [23], but oftentimes do not feel empowered due to a lack of transparency in the process, a lack of decision making power, and ambiguity about their own role in the larger organization [8, 24-25]. The engineering co- 
ops/interns from the first data set, in general, indicated clear preference for jobs and organizations in which they would feel supported and empowered to succeed. By contrast, the early career engineers from the second data set, in general, felt that they had little certainty or authority of what projects they would be working on and, seemingly, little control over their career trajectories within their firms. Similarly, the participants from the third data set spoke to changing jobs or even leaving engineering all together as their most effective tool of empowerment over the work that they would be doing. For all three data sets, this desire for involvement over the types of projects they would be working on seemed to come from a desire for greater alignment (or fit) between their personal goals, values and interests and their career lives. These results point to a tension where blue ribbon reports and professional societies and organizations make calls for skills such as innovation and creativity, leadership, and other desirable traits of engineers of the future, but it seems that engineering organizations might not be configured to allow those skills to grow and flourish. This dichotomy seems especially problematic for engineering given that women and underrepresented minorities - the very groups who might be most attracted to the field for its social good potential [26] - face yet additional barriers to staying in engineering workplaces.

\section{Interrelations between codes}

While this paper has presented data for each theme independent from one another, the authors saw significant interplay between the four elements. For example, Chris's quote under Fit also highlights the need for a mentorship structure, where he has direct access to coworkers with whom he feels comfortable approaching with questions. Here, the corporate structure was key in Chris's feelings of empowerment. Similarly, for many of the participants from the third data set, their expectations played directly into their feelings of fit with their organization. Being able to identify meaningful work within the corporate structure was key for many of these individuals to be able to find or develop personal alignment with their careers. Conversely, a lack of understanding of the corporate structure seemed to hinder participants in the second data set from developing a sense of empowerment within their jobs, and also seemed to create an atmosphere where having no expectations (or even remaining indifferent) was their best way to smoothly negotiate the transition. Future work will need to thoroughly explore the connections between these elements, possibly to find combinations that seem most effective toward improved work satisfaction and indeed, organizational structures that encourage development of skills noted as critical by reports noted above.

\section{Differences across data sets}

Although the three data sets were collected with different goals to begin with, it is important to note the ways that themes emerged both within and across data sets. There was evidence of all four themes in each data set, but the ways in which those themes were embodied were sometimes different across the entire corpus of data. For example, regarding Relational Empowerment, recent engineering graduates from Data Set 2 often discussed their jobs and positions in ways that point to a general lack of autonomy, even if they were optimistic about this changing soon. As newcomers were onboarding and adjusting to their new organizations, they tended to talk about their roles in ways that limited their control over choices and decision making. On the other hand, experienced engineers in Data Set 3 experienced higher levels of empowerment, demonstrating not only a knowledge of a wider range of choices, but also the perception that those choices were available to them. 
Similarly, participants described their Expectations in different ways across data sets. Where participants in Data Set 1 (current students) and Data Set 2 (recent graduates) had relatively few expectations, or were unsure of what their jobs would be, experienced engineers in Data Set 3 were able to articulate what they expected from their organization and describe ways to meet those expectations through intentionally working within organizational structures. Further, when discussing notions of Fit, participants described their relations with their organization differently. Whereas experienced engineers in Data Set 3 discussed fit with a more critical lens, examining the ways that their own beliefs, values, goals, and ethics could be supported by their organization, the more junior engineers in Data Sets 1 and 2 were primarily focused on fitting in as a member of their organization. It is interesting to also note that although similar themes emerged for current students, recent graduates, and more experienced practitioners, the ways in which the themes were discussed differ across participants. It seems important, then, to further explore the trajectories of engineers to better understand these "inflection points" at which they begin to more critically examine both their organization and their place within it.

\section{Conclusions and Future Work}

The purpose of this paper was to explore and reflect on the experiences of practicing engineers in the early stages of their professional careers. Through secondary analysis of interviews with engineers at three different career stages, this paper sought a better understanding of the ways in which engineering organizations enable or constrain early career engineers' sense of autonomy and empowerment in the workplace. The data showed good consistency of results across the data sets, as well as key differences between them related to the point at which participants were along their career path. In general, our results highlight some of the noted misalignments between school and work, while providing examples of where and how those misalignments are experienced by early career engineers. The findings also raise several important questions about the training and socialization of engineers, such as: how do engineers' early curricular and cocurricular educational experiences shape their expectations, and eventual experiences of the engineering workplace? In what ways are academia and industry helping engineering students develop personal goals and values, and how does this influence recent graduates' job selection, satisfaction, and persistence? And, how can corporate or organizational structures be transformed and reimagined to create workplaces in which engineers feel motivated to be change agents within their companies, and society in general, and to help make the world a better place?

To answer these questions, more studies into engineering formation and socialization are needed. The desire for alignment between personal beliefs and values, feelings of autonomy, and an empowering corporate environment are supported by the core principles of Social Cognitive Career Theory (SCCT) [27-28]. In SCCT, person-environment fit is one of the central tenants of work satisfaction. From the third data set, finding ways to integrate a notion of service into their professional engineering work was a key component for these participant's person-environment fit. Additionally, SCCT points to the need for social and material supports for an individual's central goals, which, as it relates to this study, would point to the ways in which the corporate environment listens to, recognizes, and supports the individual goals of early career engineers. For some, feeling the ability to ask questions of their boss would be one positive resource that these participants experienced towards this support. Moving forward, SCCT as well as other 
relevant theoretical frameworks should be leveraged to more deeply explore such trends and to better understand ways to support engineers as they transition from school to work as well as into their professional careers.

\section{Acknowledgments}

This paper is based on research supported by the National Science Foundation under Grants 1360665, 1360956, and 1360958 (The Professional Engineering Pathways Study; Data Set 1) and 1158863 (Engineers Views of Social Responsibility Study; Data Set 3). Any opinions, findings, and conclusions or recommendations expressed in this material are those of the author(s) and do not necessarily reflect the views of the NSF. The authors would also like to thank The Professional Engineering Pathways Study research team (Holly Matusovich, Ruth Streveler, Sheri Sheppard, Cheryl Carrico, and Helen Chen), Marie Paretti, and Angela Bielefeldt for their support and collaboration on the research projects described within.

\section{References}

[1] National Academy of Engineering, Educating the Engineer of 2020: Adapting Engineering Education to the New Century. Washington, D.C.: National Academies Press, 2005.

[2] G. W. Clough, The Engineer of 2020: Visions of Engineering in the New Century. Washington, D.C.: National Academies Press, 2004.

[3] ASME Center for Education, Vision 2030: Creating the Future of Mechanical Engineering Education, Phase 1 Final Report. Washington, D.C.: American Society of Mechanical Engineers, 2011.

[4] ASCE Body of Knowledge Committee, Civil Engineering Body of Knowledge for the 21 st century: Preparing the Civil Engineer for the Future. Washington, D.C.: American Society of Chemical Engineers, 2008.

[5] R. L. Collins, "Engineering graduate preparedness for the workplace: Employer assessments of outcome based education," Ph.D. dissertation, University of Nevada, Reno, Nevada, 2008.

[6] J. L. Sussman, P. Brackin, and S. A. Rajala, "ABET update: Proposed revisions to EAC General Criteria 3 and 5," presented at the American Society for Engineering Education (ASEE) Engineering Dean's Institute, San Francisco, CA, 2016.

[7] R. Korte, "Learning how things work here: The socialization of newcomers in organizations," in Supporting Workplace Learning: Towards Evidence-Based Practice, Professional and Practice-Based Learning, vol. 5, R. F. Poell and M. van Woerkom, Eds., Dordrecht, The Netherlands: Springer, 2011, pp. 129-146.

[8] R. Korte, S. Brunhaver, and S. Sheppard, "(Mis) Interpretations of organizational socialization: The expectations and experiences of newcomers and managers," Hum Resource Dev Q, vol. 26, no. 2, 185-208, 2015.

[9] J. Trevelyan, "Reconstructing engineering from practice," Eng Stud, vol. 2, no. 3, 175 $195,2010$.

[10] J. Trevelyan, "Understanding the technical and the social," in Engineering Practice in a Global Context, B. Williams, J. Figueiredo, and J. Trevelyan, Eds., Leiden, The Netherlands: CRC Press, 2013, pp. 33-60. 
[11] B. D. Lutz, "Into the workplace: Exploring the learning experiences of newcomer engineers during the school-to-work transition," Ph.D. dissertation, Virginia Polytechnic Institute and State University, Blacksburg, VA, 2017.

[12] V. Braun and V. Clarke, "Using thematic analysis in psychology," Quant Res Psych, vol. 3, no. 2, 77-101, 2006.

[13] J. Heaton, "Secondary analysis of qualitative data: An overview," Hist Soc Res, vol. 33, no. 3, 33-45, 2008.

[14] S. Brunhaver, R. Streveler, C. Carrico, H. Matusovich, P. Boylan-Ashraf, and S. Sheppard, "Professional Engineering Pathways Study: A longitudinal study of early career preparedness and decision-making," in Proc Front Educ Ann Conf, 2015.

[15] R. Abhyankar, C. Carrico, H. M. Matusovich, and S. Brunhaver, "Junior and senior engineers' beliefs about the influence of socializers on their first job-related decisions," in Proc Am Soc Eng Educ Ann Conf, 2018.

[16] N. Canney, "Shaping future engineers through service learning in engineering education," in Engineering Professionalism - Engineering Practices in Work and Education, S. Brodersen, and U. Jørgensen, Eds., Rotterdam, The Netherlands: Sense Publishers.

[17] A. Bielefeldt, and N. Canney, "Working engineers' satisfaction with helping people and society through their jobs," Eur J Eng Educ, DOI: 10.1080/03043797.2018.1476468.

[18] R. Stevens, K. O'Connor, L. Garrison, A. Jocuns, and D. M. Amos, "Becoming an engineer: Toward a three dimensional view of engineering learning," J Eng Educ, vol. 97, no. 3, 355-368, 2008.

[19] K. D. O’Connor, D. Amos, T. Bailey, L. Garrison, H. Loshbaugh, M. Jones, D. Seward, L. Perhamus, and R. Stevens, "Sponsorship: Engineering's tacit gatekeeper," in Proc Am Soc Eng Educ Ann Conf, 2007.

[20] L. Geppert, "Educating the renaissance engineer," IEEE Spec, vol. 32, no. 9, 39-43, 1995.

[21] M. Jones, "The renaissance engineer: A reality for the 21 st century?," Eur J Eng Educ, vol. 28, no. 2, 169-178, 2003.

[22] G. Lewis, "Why Being Vulnerable at Work Can be Your Biggest Advantage, according to Brene Brown," business.linkedin.com, October 4, 2017. [Online]. Available: https://business.linkedin.com/talent-solutions/blog/talent-connect/2017/why-beingvulnerable-at-work-can-be-your-biggest-advantage-according-to-brene-brown

[23] M. Klenk, T. Bjorklund, S. Gilmartin, and S. Sheppard, "Early-career engineers at the workplace: Meaningful highs, lows, and innovative work efforts," in Proc Am Soc Eng Educ Ann Conf, 2018.

[24] R. Korte, W. Jordan, and S. Sheppard, "A qualitative study of the early work experiences of recent graduates in engineering," in Proc Am Soc Eng Educ Ann Conf, 2008.

[25] S. Brunhaver, R. Korte, M. Lande, and S. Sheppard, "Supports and barriers that recent engineering graduates experience in the workplace," in Proc Am Soc Eng Educ Ann Conf, 2010.

[26] National Academy of Engineering, Committee on Public Understanding of Engineering Messages. Changing the conversation: Messages for improving public understanding of engineering, Washington, D.C.: National Academies Press, 2008.

[27] R. W. Lent, S. D. Brown, and G. Hackett, "Toward a unifying social cognitive theory of career and academic interest, choice, and performance," J Voc Behav, vol. 45, no. 1, 79122. 
[28] R. W. Lent and S. D. Brown, "Integrating person and situation perspectives on work satisfaction: A social-cognitive view," J Voc Behav, vol. 69, no. 2, 236-247. 